\title{
Suppressor role of androgen receptor in proliferation of prostate basal epithelial and progenitor cells
}

\author{
Soo Ok Lee ${ }^{1, *}$, Jing Tian ${ }^{1,2, *}$, Chiung-Kuei Huang ${ }^{1, *}$, Zhifang Ma ${ }^{1}$, Kuo-Pao Lai ${ }^{1}$, HsiMin Hsiao ${ }^{1}$, \\ Ming Jiang ${ }^{3}$, Shuyuan Yeh ${ }^{1}$ and Chawnshang Chang ${ }^{1,4}$ \\ ${ }^{1}$ George Whipple Laboratory for Cancer Research, Departments of Pathology, Urology, Radiation Oncology, and the Wilmot Cancer Center, University of \\ Rochester Medical Center, Rochester, New York 14642, USA \\ ${ }^{2}$ Institute of Urology, Chawnshang Chang Sex Hormone Research Center, 2nd Affiliated Hospital of Tianjin Medical University, Tianjin, \\ People's Republic of China \\ ${ }^{3}$ Department of Urologic Surgery, Vanderbilt University Medical Center, Nashville, Tennessee 37232, USA \\ ${ }^{4}$ Sex Hormone Research Center, China Medical University/Hospital, Taichung 404, TN, Taiwan \\ (Correspondence should be addressed to S O Lee; Email: soook_lee@urmc.rochester.edu; C Chang at George Whipple Laboratory \\ for Cancer Research, University of Rochester Medical Center; Email: chang@urmc.rochester.edu) \\ *(S O Lee, J Tian and C-K Huang contributed equally to this work)
}

\begin{abstract}
Early studies have reported the differential roles of androgen receptor (AR) in different types (luminal, basal intermediate, and stromal) of prostate cancer cells. In vivo mouse model tumor studies using the total prostate epithelial knockout mice (pesARKO) also revealed that AR played a suppressive role in proliferation of the $\mathrm{CK} 5{ }^{+} / \mathrm{CK} 8{ }^{+}$progenitor/intermediate cells but a positive role in the $\mathrm{CK} 5^{-} / \mathrm{CK} 8^{+}$luminal epithelial cells. Using three different resources (one human basal epithelial cell line, one mouse basal epithelial originated progenitor cell line, and a basal epithelium-specific ARKO mouse model), we here demonstrated that the AR in basal epithelial cells of normal
\end{abstract}

prostate plays a suppressive role in their proliferation but a positive role in differentiation into luminal epithelial cells. These results led us to conclude that ARs may play a negative role to suppress $\mathrm{CK}^{+}{ }^{+}$basal epithelial and progenitor cell proliferation, yet play an essential role to drive basal epithelial cells into more differentiated states. These results may explain why differential AR expression in different cell types within normal prostate is needed and suggest that ARs in prostate basal epithelial cells, although expressed at a very low level, are necessary to maintain the balance between progenitor cells and differentiated luminal epithelial cells.

Journal of Endocrinology (2012) 213, 173-182

\section{Introduction}

Normal adult prostatic epithelium includes three types of cells: neuroendocrine cells, luminal epithelial cells, and basal epithelial cells that are aligned along the basement membrane. Cells in the luminal layer express androgen receptor (AR), prostate-specific antigen (PSA), cytokeratin8 (CK8), and CK18. In contrast, cells of the basal layer express CK5 and CK14 (Verhagen et al. 1988, Hayward et al. 1996, Xue et al. 1998a, Johnson et al. 2000), and AR expression is either undetectable (Prins et al. 1991, Uzgare et al. 2004) or at a very low level (Bonkhoff et al. 1998). More than $80 \%$ of proliferating cells are observed in the basal layer, which is thought to contain $\mathrm{CK} 5^{+} / \mathrm{CK} 8^{-}$stem cells that give rise to $\mathrm{CK} 5^{+} / \mathrm{CK} 8^{\text {low }}$ progenitor/intermediate cells and can then be further differentiated into the secretory luminal $\mathrm{CK} 5^{-} / \mathrm{CK}^{\text {high }}$ cells (Bonkhoff et al. 1994, Xue et al. 1998b).

Under normal conditions, adult prostate epithelial cells remain in homeostasis with infrequent turnover. However, the homeostasis is broken upon androgen depletion. When an adult male is castrated, the serum testosterone level decreases rapidly and the prostate regresses with the majority of luminal epithelial cells dying through apoptosis, whereas the majority of basal epithelial cells remain alive (Bonkhoff et al. 1994). These contrasting results suggest that response to androgen deprivation treatment is different in normal luminal epithelial cells vs basal epithelial cells.

Using established mouse and human basal epithelial/progenitor cells as well as an in vivo basal-ARKO (Cyp19a1) mouse model, we report that ARs may play a negative role to suppress proliferation of basal epithelial/progenitor cells, yet AR expression also plays essential roles to drive these cells into a more differentiated state.

\section{Materials and Methods}

Development of basal-ARKO mice

All research was approved and conducted following the rules/regulations of the Institutional Review Board (IRB) and University Committee of Animal Research (UCAR), 
which was fully credited by AAALAC (ID: A-3292-01), at the University of Rochester Medical Center. The generation of basal-ARKO mice was carried out by mating female mice harboring floxed AR alleles with male transgenic mice expressing Cre recombinase under the control of the CK5 promoter. The detailed strategies of mating and genotyping were described (Yeh et al. 2002). Basal-ARKO mice carrying floxed $A R$ and Cre were selected after genotyping.

\section{Histological examination of mice}

Briefly, mice were killed using $\mathrm{CO}_{2}$. Ventral prostate (VP), anterior prostate (AP), and dorsal lateral prostate were removed, fixed in 10\% neutral buffered formalin, and subjected to paraffin sectioning. Tissue sections from $\mathrm{VP}$ at different ages were stained with hematoxylin and eosin and their morphology examined. The images were acquired using an E800 microscope (Nikon) and a SPOT camera (Diagnostic Instruments Sterling Heights, MI, USA) and were analyzed using SigmaScan Pro Sysat software (version 5.0; SPSS Inc., Chicago). For staining of tissues, antibodies of AR (1:200, N20; Santa Cruz, Biotechnologies, Santa Cruz, CA, USA), p63 (1:200; Abcam, Cambridge, UK), Ki67 (1:200; Novocastra, Newcastle, UK), CK5 (1:250; Covance, Princeton, NJ, USA), and CK8 (1:250; Abcam) were used.

\section{Cell culture}

Cultures of mouse prostate epithelial cells (mPrE) were maintained in RPMI medium containing 10\% fetal bovine serum (FBS). Normal human basal cells from Lifeline Cell Technology (Lifeline-basal) were maintained in ProstaLife prostate epithelial cell culture medium (Lifeline, Frederick, MD, USA) supplemented with LifeFactors (transforming growth factor- $\alpha$, epinephrine, insulin, transferrin, and hydrocortisone) provided with medium.

\section{MTT cell growth assay}

Cells were plated onto 24-well plates. At various time points indicated, MTT solution was added onto cells for $30 \mathrm{~min}$, media were removed, isopropanol was used to dissolve the MTT salt, and ODs were measured at $570 \mathrm{~nm}$.

\section{Immunohistochemical staining/immunofluorescence staining of cells/tissues}

Cells were seeded on four-well chamber slides and fixed with methanol. After fixation, cells were washed with PBS three times for $5 \mathrm{~min}$, then cells were blocked with $1 \%$ FBS for $1 \mathrm{~h}$, washed with PBS three times, and then incubated with primary antibodies in 3\% BSA in PBS overnight at $4{ }^{\circ} \mathrm{C}$. Antibodies used were as follows: anti-Ki67 (1:1000; National Chemical Lab, Pune, India), anti-p63 (1:250; Santa Cruz), anti-AR (1:250; N20, Santa Cruz), anti-PSCA (1:200; N20, Santa Cruz), anti-CD44 (1:250; N20, Santa Cruz), anti-CK5 (1:250;
Covance), anti-CK8 (1:250; Abcam), and anti-sca-1 (1:250; eBioscience, San Diego, CA, USA). Cells were then incubated with 1:200 diluted biotinylated secondary antibody (Vector Laboratories, Burlingame, CA, USA) and ABC solution (Vector Laboratories) for immunohistochemistry (IHC), or with fluorescent secondary antibodies for immunofluorescence (IF) (either Alexa 594 or Alexa488 tagged). For IHC, cells were stained by AEC (Dako, Carpenteria, CA, USA), followed by Mayor's hematoxylin counterstaining.

Formalin-fixed paraffin wax-embedded tissues were cut into $5 \mu \mathrm{m}$ sections, placed on poly-L-lysine-coated slides, deparaffinized, rehydrated, subjected to antigen retrieval for $10 \mathrm{~min}$, blocked with normal horse serum, and incubated with appropriate primary monoclonal antibodies, appropriate fluorescein-tagged secondary antibody (for IF), or visualized by VECTASTAIN ABC peroxidase system and diaminobenzidine (DAB) kit (Vector Laboratories) (for IHC).

\section{Self-renewal analysis}

Single cell suspension $\left(1 \times 10^{3}\right.$, in $50 \mu 1$ medium) was mixed with $50 \mu \mathrm{l}$ cold Matrigel and the mixture was placed along the rim of the 24 wells with minimum of triplicate experiments. The culture plates were placed in a $37{ }^{\circ} \mathrm{C}$ incubator for $10 \mathrm{~min}$ to let the mixture solidify and $500 \mu \mathrm{l}$ medium was then added into the well. Cells were grown in culture media and sphere numbers were counted after 7-14 days.

\section{Western blot analysis}

Cell lysates were resolved with $10 \%$ SDS gel electrophoresis (SDS-PAGE), blotted with AR antibody (N20; Santa Cruz), and incubated with secondary antibodies conjugated with horseradish peroxidase. Proteins were visualized using the Pierce ECL Western Blotting Substrate (Thermo-Fisher Scientific, Rochester, NY, USA) according to the manual's instructions. Glyceraldehyde 3-phosphate dehydrogenase (GAPDH) served as loading controls.

\section{RNA extraction and real-time quantitative PCR (qRT-PCR)}

Total RNAs were extracted with Trizol (Invitrogen). mRNAs $(2 \mu \mathrm{g})$ were used for reverse transcription to cDNAs using Superscript III (Invitrogen). Quantitative qRT-PCR was performed using cDNA, specific gene primers, and SYBR green master mix (Bio-Rad) on an iCycler iQ Multi-color real-time PCR machine (Bio-Rad).

\section{Flow cytometry analysis of cell markers}

Cells were detached with $5 \mathrm{mM}$ EDTA and washed with $1 \%$ FBS in PBS (flow washing buffer); stained with sca-1, CD49f, and CD44 (eBioscience), washed again with 1\% FBS in PBS, resuspended in PBS; and then analyzed by flow cytometry according to the previous publication (Lai et al. 2009). 


\section{Lentivirus infection}

$293 \mathrm{~T}$ cells were transfected with a mixture of DNAs (Lentiviral vectors pWPI) (Addgene, Cambridge, MA, USA) containing AR (vectors used as controls), pMD2.G packaging plasmid, and psPAX2 envelope plasmid (4:1:1 ratio) using Lipofectamine (Invitrogen) according to the transfection protocol. Culture media containing virus was collected $32 \mathrm{~h}$ after transfection, filtered, and added onto cultured cells with $2 \mu \mathrm{g} / \mathrm{ml}$ polybrene. Cells were refreshed with culture media $24 \mathrm{~h}$ later and cultured for 3 days. As the pWPI vector contains green fluorescent protein (GFP), transfection efficiency was monitored by fluorescence microscopy.

\section{Apoptosis assay}

The in situ cell death detection kit (Roche Pharmaceuticals) was used according to the manufacturer's instruction for detection of apoptotic cells.

\section{$\beta$-Galactosidase assay}

Six weeks after mating of CK5-Cre/floxed AR and ROSA26, prostate lobes were taken, processed, and embedded with OCT media. The $5 \mu \mathrm{m}$ sections were used to perform the $\beta$-galactosidase assay according to the manufacturer's instruction.

\section{Luciferase assay}

Cells were plated in 24-well plates and transfected with MMTV-luc containing androgen response element (ARE) sequence using Lipofectamine (Invitrogen) according to the manufacturer's instruction. After transfection, RPMI medium containing charcoal-stripped medium (for $\mathrm{mPrE}$ ) and basal cell medium (without serum, for Lifeline-basal cells) was added into the culture with addition of various concentrations of dihydrotestosterone (DHT), 0 (ethanol as vehicle control), 1, and $10 \mathrm{nM}$, and incubated for $48 \mathrm{~h}$. pRL-TK was used as internal control. Luciferase activity was measured by DualLuciferase Assay (Promega) according to the manufacturer's manual.

\section{Statistical analysis}

Values were expressed as mean \pm s.D. The Student's $t$-test was used to calculate $P$ values. $P<0 \cdot 05$ was considered significant.

\section{Results}

\section{AR suppresses growth of prostate basal epithelial and progenitor cells}

To investigate the potential role of AR in proliferation of prostate basal epithelial and progenitor cells, we selected two established cell lines, human basal epithelial cell line (named Lifeline-basal) and a mouse progenitor cell line $(\mathrm{mPrE})$. Figure $1 \mathrm{~A}$ includes results of various studies using the $\mathrm{mPrE}$ cell line whereas Fig. 1B shows results using the Lifeline-basal cell line.

The $\mathrm{mPrE}$ cell line was originally derived from normal mouse prostate tissues (Wang et al. 2000). The morphology of $\mathrm{mPrE}$ cells is shown in Supplementary Figure 1A, see section on supplementary data given at the end of this article. The initial examination of characteristics of these cells revealed that they are of basal epithelial origin (Wang et al. 2000) and the IF staining result showed positive staining with basal cell markers CK5 and p63 (Supplementary Figure 1B). The flow cytometric analysis result (Supplementary Figure 1C) further demonstrated that $98 \%$ of these cells stained positive for stem/progenitor markers, sca-1 and CD49f, whereas the immunoglobulin G control showed almost no signals for sca-1 and CD49f. These cells were not positively stained for the intermediate cell marker, prostate stem cell antigen (data not shown). Together, these results indicate that the $\mathrm{mPrE}$ cells are basal epithelial originated progenitor cells. These $\mathrm{mPrE}$ cells express little AR as shown in western blot (Fig. 1A-a), qRT-PCR (Fig. 1A-b), and IF staining (Fig. 1A-d) analyses. To investigate whether the low level of AR is critical in self-renewal/proliferation of these cells, we forcedly expressed AR into the cells via viral infection and MTT assay, sphere formation assay, and Ki67 labeling experiments were performed. Interestingly, the proliferation of the $\mathrm{mPrE}$ cells was shown to be significantly suppressed upon AR expression (MTT assay result at day 6, Fig. 1A-c, and Ki67 IF staining result, Fig. 1A-d), indicating that $A R$ plays a negative role to suppress their proliferation. The MTT assay was performed at 0 (vehicle control), 1 (the prostate intra tissue DHT level after castration) (Titus et al. 2005a,b), and $10 \mathrm{nM} \mathrm{DHT} \mathrm{(the} \mathrm{prostate} \mathrm{intra-tissue}$ DHT level before castration), and the suppressive effect of AR on proliferation was observed at all androgenic concentrations. Selfrenewal tests were also performed and the results showed that the AR-expressing $\mathrm{mPrE}$ cells have lesser self-renewal ability compared with control cells (Fig. 1A-e). All these results indicate the suppressive role of $\mathrm{AR}$ in self-renewal/proliferation of $\mathrm{mPrE}$ progenitor cells.

The Lifeline-basal cells (also named as HPrE), which show characteristics of basal epithelial cells, were originally derived from a normal human donor. These cells were also used in our study to investigate the AR effects on proliferation of basal cells. The morphology of these cells is shown in Supplementary Figure 2A, see section on supplementary data given at the end of this article. These human basal cells showed positive staining with CK5 and p63 but failed to express CK8 (Supplementary Figure 2B). Similar to the $\mathrm{mPrE}$ cell line data, a low expression of AR was observed (protein level in Fig. 1B-a and mRNA level in Fig. 1B-b). We also tested whether the proliferation of these cells was also suppressed upon addition of AR. As shown in Fig. 1B-c, the addition of AR suppressed cell growth at all androgen concentrations tested starting on day 3. IF staining analysis result showing lower numbers of Ki67 positively stained cells upon AR addition (Fig. 1B-d) also indicated that AR plays a suppressive role in proliferation of these basal epithelial cells. 
A mPrE cell line

(a)

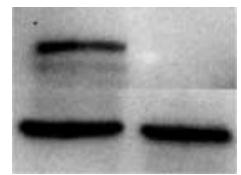

LNCaP mPrE

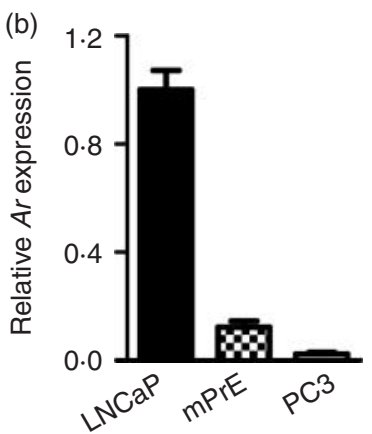

B

(a)

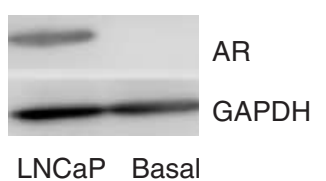

(b)

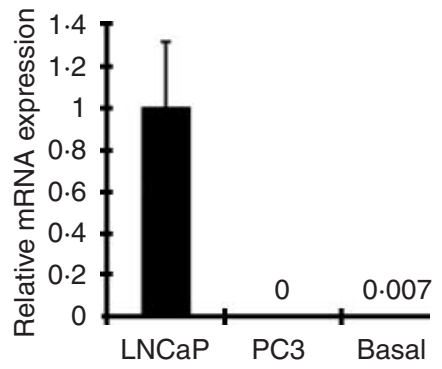

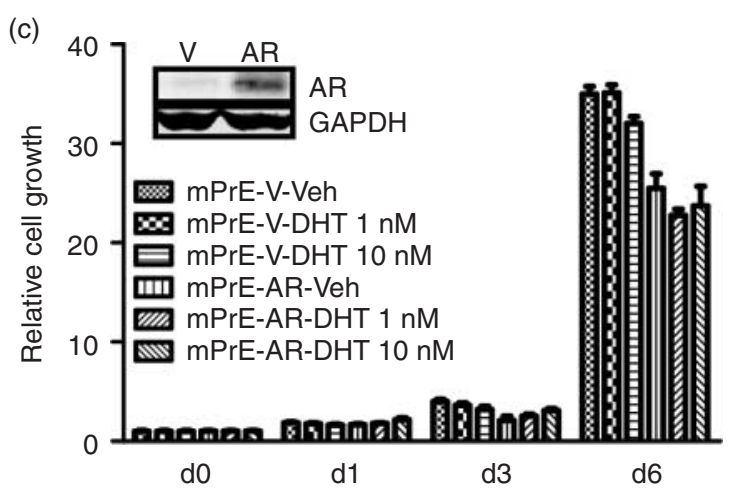

(d)
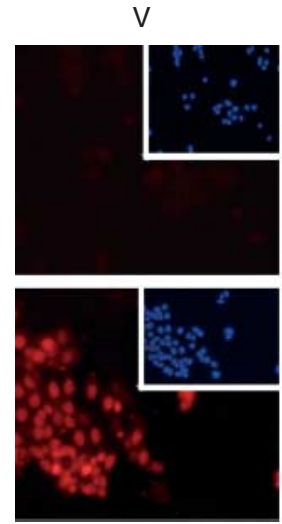

AR
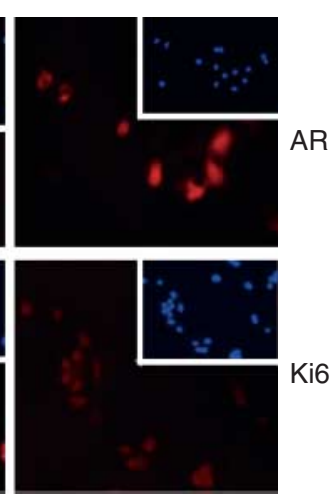

(c)

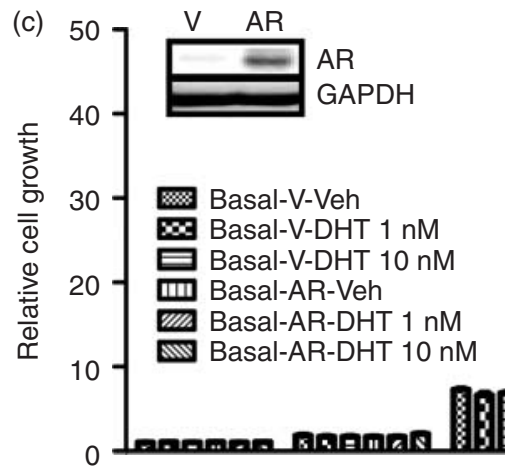

d0

(d)
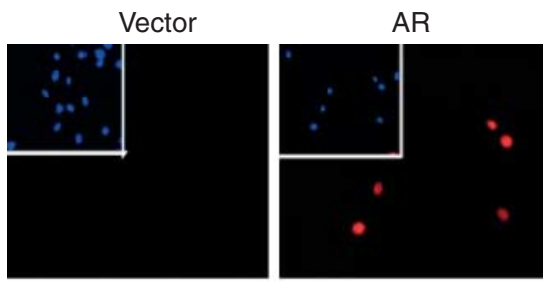

AR
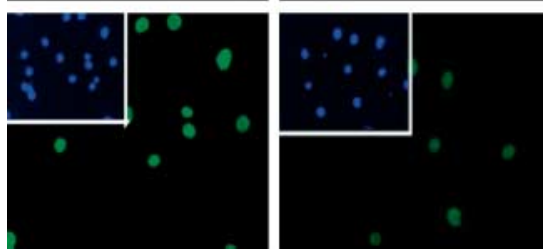

(e)
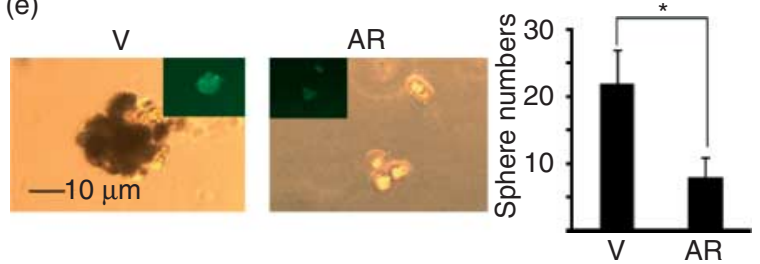

Figure 1 AR expression suppresses self-renewal/proliferation of $m \operatorname{PrE}(A)$ and Lifeline-basal epithelial cells (B). (a) Western blot analysis of AR expression. Cell extracts were obtained from mPrE/Lifeline-basal cells and LNCaP cells (used as positive control) and analyzed by western blot analysis. (b) qRT-PCR analysis results of AR mRNA expression. The mRNAs of mPrE/Lifeline-basal cells were analyzed by qPCR analysis. The human prostate cell lines LNCaP and PC3 were used as positive and negative controls respectively. (c) MTT assays at three different DHT concentrations, 0 (vehicle control), 1, and $10 \mathrm{nM}$, are shown. Western blot results are shown to indicate

AR overexpression. (d) Ki67 IF staining results after infection of cells with lentivirus carrying either vector or AR. After infection, cells were plated and immunofluorescence (IF) staining was performed using Ki67 antibody. AR staining presents different levels of AR expression after the infection. (A-e) Self-renewal test on Matrigel after infection of mPrE cells with lentivirus carrying either vector or AR. Quantitation shown at right. ${ }^{*} P<0.03$. 
Mice lacking $A R$ in basal epithelial cells led to increased proliferation of $\mathrm{CK}^{+}$cells

In our earlier studies, the increase in $\mathrm{CK}^{+}$cells was also detected when AR was knocked out in prostate epithelium in TRAMP derivatives of prostate epithelium-specific ARKO (pes-ARKO-TRAMP) mice (Niu et al. 2008). As an extension of this finding and our in vitro results shown in Fig. 1B, we tried to elucidate the significance of the low level of AR in basal epithelial cells, although the endogenous AR level is very low. We developed a mouse model in which AR is specifically knocked out in basal epithelial cells (basalARKO) by mating male CK5-Cre mice with female floxAR mice (Yeh et al. 2002). The Supplementary Figure 3A, see section on supplementary data given at the end of this article, shows the strategy for developing basal-ARKO mice and Supplementary Figure 3B genotyping shows Cre and floxAR expressions in transgenic mice. We bred ROSA26R mice with the CK5-Cre, floxed AR/AR mice to monitor the gene deletion efficiency. In the ROSA26R mice, generalized transcription of the $\beta$-gal (Glb1) gene is prevented by a preceding transcriptional stop sequence, which is flanked by two loxP sites. In crosses of ROSA26R transgenic mice with promoter-derived Cre transgenic mice, the transcriptional stop sequence was removed by Cre-mediated excision and GLB1 protein will be expressed (Soriano 1999). As shown in Fig. 2A, we observed blue staining in almost all basal epithelium areas, but not in luminal epithelium, suggesting basal epithelium-specific AR knockout. The blue staining was more obvious in VP and dorsolateral prostate lobes than AP lobe (data not shown). To further verify that AR was knocked out in basal epithelium but not in luminal epithelium, we performed IF double staining of VPs of 4-week-old wild-type (WT) and basal-ARKO mice using antibodies of basal cell marker, p63, and AR (Fig. 2B). The AR/p63 positively stained cells (Fig. 2B, shown in yellow, arrows) were detected in WT mice but not in basal-ARKO mice (Fig. 2B, shown in red). However, strong AR staining (green) was detected in luminal epithelium of both WT and basal-ARKO mice. These results indicate that AR was specifically knocked out in basal epithelium in the basalARKO mice. The AR knockout efficiency was more than $80 \%$ when judged by the AR/p63 IF double staining. The AR knockout in basal epithelium was more obvious in younger (4 and 6 weeks) mice compared with older mice (data not shown) indicating that the knockout effect is maximal at this age and this AR knockout effect might be compensated for in old mice by other factors.

We found that total body weight (data not shown) and testosterone level (Supplementary Figure 3C) were similar between WT and basal-ARKO mice, although some disorganized membrane structures between epithelium and stromal compartment in prostate glands of 4- and 6-week-old basalARKO mice were detected (Supplementary Figure 3D, arrows). Similar phenotypes of membrane disorganization were reported when p63 was depleted from the basal cell layer (Liu et al. 2009).
They suggested that this phenotype could promote tumor invasion and progression, but whether this phenotype is correlated with any function and any other phenotype remains unclear.

We analyzed the distribution of $\mathrm{CK}^{+}{ }^{+}$and $\mathrm{CK}^{+}$cells in VP tissues of basal-ARKO and control WT littermate mice (4 weeks old) and found that the $\mathrm{CK}^{+}{ }^{+}$cells (basal epithelial including stem/progenitor/intermediate cells) (Fig. 2C, stained green, arrow) were significantly increased in VPs of 4-week-old basal-ARKO mice compared with WT control mice. The increased $\mathrm{CK}^{+}$cell numbers could be due to higher proliferation and/or lower apoptotic death. We could detect significantly higher numbers of Ki67 positively stained cells in 4- and 6-week-old basal-ARKO mice compared with WT mice (Fig. 2D). In contrast, we failed to observe significant differences in apoptotic death rate between these two types of mice (data not shown). These results indicate that AR plays a negative role to suppress proliferation of basal cells, which is in agreement with the in vitro studies previously shown (Fig. 1).

\section{$A R$ role in basal epithelial cells in their differentiation process}

We found that AR knockout in basal epithelial cells promoted their proliferation (Figs 1 and 2). We next investigated whether AR in basal epithelial cells plays a role in the differentiation process into terminally differentiated luminal epithelial cells by exploiting the basal-ARKO mice model. We generated CK5-Cre/WTAR:ROSA26 and CK5-Cre/ floxAR:ROSA26 mice by crossing the CK5-Cre/WT AR and CK5-Cre/floxAR mice with the ROSA26 mice respectively. We found that GLB1 activity (blue color) was expressed in all prostate tissues including luminal epithelium in the CK5-Cre/WTAR:ROSA26, indicating that the GLB1 activity is from the AR-containing basal epithelial cells. However, the GLB1 activity was detected only in basal epithelium in CK5-Cre/floxAR:ROSA26 mice and the luminal epithelium did not show blue color (Fig. 3A). It may be that the AR knocked out basal epithelial cells in the CK5-Cre/floxAR:ROSA26 mice were not able to differentiate into luminal epithelial cells, so blue staining was not observed. The luminal epithelium was probably due to the incomplete AR knockout in $20 \%$ of basal epithelial cells. These results indicate that basal AR, although the level is very low, is essential to drive cells to differentiate.

The AR role in basal cell differentiation has also been examined in $\mathrm{mPrE}$ and Lifeline-basal cells at three androgenic concentrations. When these cells were transiently transfected with AR, the increase in expressions of PSA and the differentiation markers, CK8 and Nkx3.1 (Gurel et al. 2010), was detected while the expression of CK5 was decreased, which also suggests that AR expression drives basal epithelial cells toward the differentiated state (Fig. 3B).

Finally, we investigated the mechanism by which the AR introduction inhibited self-renewal/proliferation of the progenitor/basal epithelial cells. After we introduced AR by transient transfection into both $\mathrm{mPrE}$ and Lifeline-basal cells, 
A

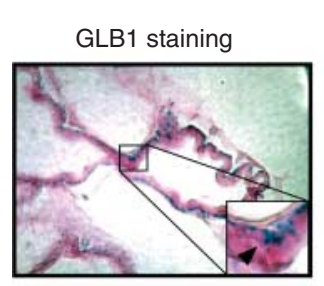

C
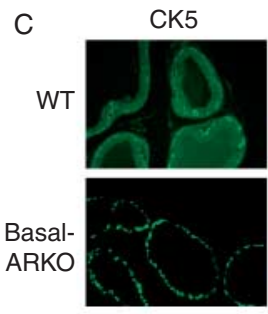

D
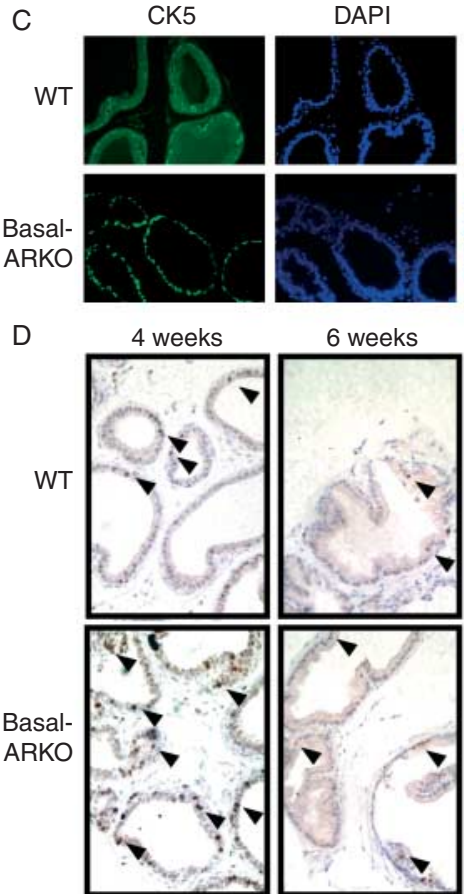

6 weeks
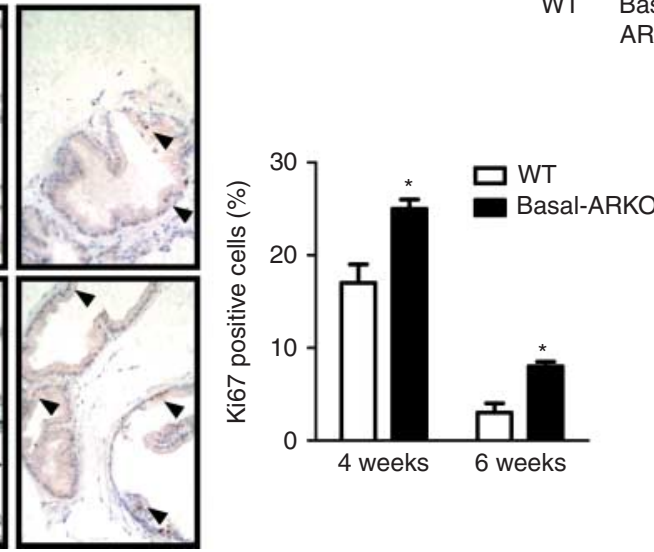

Figure 2 CK5-positive cells are increased in basal-ARKO mice compared with WT control mice. (A) GLB1 activity (blue color) was shown in CK5-Cre mice crossed with ROSA26 mice. Arrowhead points out positive basal cell layer. (B) IF staining of AR/p63 in VP tissues obtained from 4-week-old WT and basal-ARKO mice. The green and red signals represent AR and p63 staining, respectively, and the yellow staining indicates double positively stained cells (arrows). The double positively stained cells for AR/p63 were high in WT mice but were decreased in basal-ARKO mice and the AR-positive cells were shown in luminal epithelium part only. (C) CK5 IF staining result of VPs of 4-weekold WT and basal-ARKO mice. Basal-ARKO mice showed higher CK5-positive cells compared with the WT mice indicating expansion of CK5-positive cells upon AR knockout. Right panel shows ratio of quantification of CK5-positive signals/DAPI nuclear signal. ${ }^{* * *} P<0.009$ (D) Ki67 IHC staining result of VPs of WT and basal-ARKO mice at 4 and 6 weeks. The Ki67 positively stained cells (arrow heads) were increased in basal epithelium in basal-ARKO mice compared with the WT mice. Quantitation result shown at right *(4 weeks) $P<0.04 ;{ }^{*}(6$ weeks) $P<0.025$. the transactivation of AR was confirmed by luciferase assay using MMTV-luciferase including ARE. As shown in Fig. 4A and $B$, the luciferase activity was increased upon $A R$ transfection, confirming that the transfected AR was functional. Then the expression/activation of several signaling pathways and anti-apoptotic protein in $\mathrm{mPrE}$ and Lifeline-basal cells was analyzed by western blot analysis. We found decreases in AKT (AKT1) signal (shown by p-AKT level) and BCL2 expression in both cell lines, whereas no difference was detected in other signals including ERK (MAPK1), WNT (WNT2), and
c-MYC (MYC) (Fig. 4C and D). These results suggest that AKT signal and BCL2 are critical in promoting self-renewal/ proliferation of these cells.

\section{Discussion}

Earlier in vitro studies using different prostate epithelial cell lines led to different conclusions that androgen might be able to either stimulate or suppress prostate epithelial cell proliferation 
A

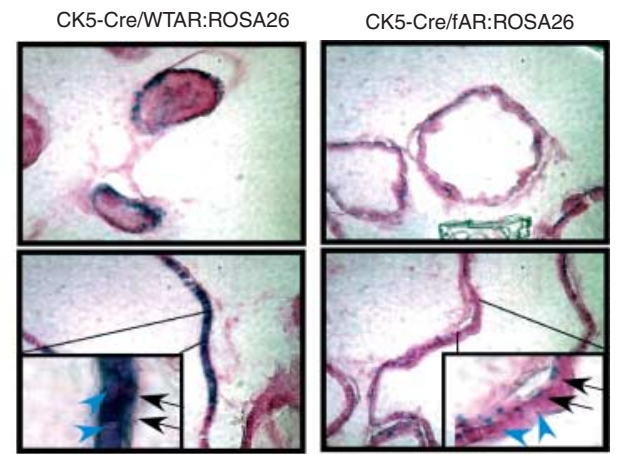

B
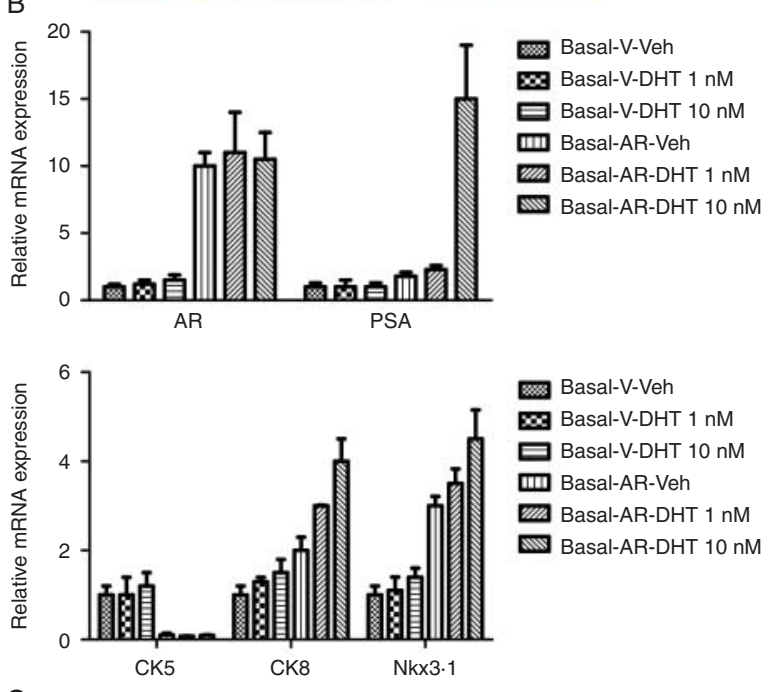

C

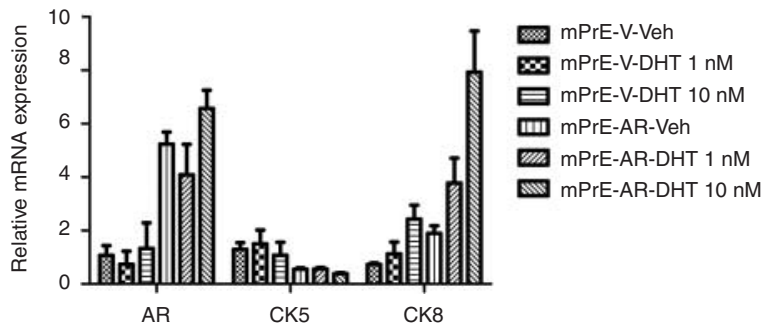

Figure 3 AR expression induces differentiation of basal epithelial cells. (A) CK5-Cre/WTAR:ROSA26 and CK5-Cre/floxAR:ROSA26 mice were generated by crossing the CK5-Cre/WT AR and CK5-Cre/floxAR mice with the ROSA26 reporter mice respectively. The mice were killed at 6 weeks of age and GLB1 staining in VP was monitored. GLB1 activity was shown in blue staining and insets represent magnified staining. Arrows indicate luminal cells and arrowheads point out basal cell layer. (B) The mPrE and (C) Lifelinebasal cells were infected with lentivirus carrying AR/vector and grown in three different androgenic concentrations $(0,1$, and $10 \mathrm{nM})$, and the expressions of differentiation markers, PSA, CK8, and Nkx3.1, were examined together with basal marker, CK5, by qRT-PCR analysis.

(Nishi et al. 1988, Altuwaijri et al. 2007, Simanainen et al. 2009, Liao et al. 2010). However, which type of prostate epithelial cells could be suppressed by androgen remained unclear, especially when using in vivo animal models to prove which epithelial cell type of prostate could be stimulated or suppressed by androgen.
Using orthotopic injection of $\mathrm{CK}^{+}$basal intermediate type of human prostate cancer (PCa) PC3 cells, it was demonstrated that PC3 tumor growth was inhibited when AR was introduced (Niu et al. 2008). The increase in $\mathrm{CK} 5^{+}$ cells was also detected when AR was knocked out in prostate epithelium in TRAMP derivatives of prostate epitheliumspecific ARKO (pes-ARKO-TRAMP) mice (Niu et al. 2008). These results indicated that AR might play a suppressive role in proliferation of $\mathrm{PCa} C K 5^{+}$cells. Recently, we have also found that the AR role in self-renewal/ proliferation of the $\mathrm{CK} 5^{+} \mathrm{PCa}$ stem/progenitor cells is opposite (suppressor) to the stimulator AR role in proliferation of the $\mathrm{CK}^{+}$nonstem/progenitor cells (Lee et al., manuscript submitted). Here, we found similar results showing the suppressor role of AR in self-renewal/ proliferation of the normal prostate $\mathrm{CK} 5^{+}$progenitor and basal epithelial cells (Figs 1 and 2).

The androgen/AR roles in differentiation of prostate epithelium have been extensively studied (Ling et al. 2001, Whitacre et al. 2002, Garraway et al. 2003, Berger et al. 2004). When the AR gene was selectively knocked out in epithelial cells, the AR-deficient cells did not terminally differentiate (Simanainen et al. 2007, 2009), which impedes prostate lobes' growth and function (Wu et al. 2007). However, these results were due to the AR knockout in total epithelium, and up-tonow no basal epithelium-specific AR knockout mouse models have been developed and thus the AR role in basal epithelium has not been studied extensively.

The indication of importance of very low AR expression levels in basal epithelial cells is of significance. The very low levels of AR in the basal compartment may be important in providing a good environment for stem/progenitor cells' self-renewal/proliferation. However, the AR in basal epithelial cells enables these cells to differentiate into luminal epithelial cells (Fig. 4A), and we have observed higher expression of differentiated cell markers upon AR expression (Fig. 4B and C). So it can be speculated that maintaining the low level of AR in basal epithelial cells might be essential to maintain the balance between progenitor and differentiated luminal epithelial cells and total lack of AR in basal epithelial cells might impede their ability to differentiate.

Taken together, we conclude that the low level of AR expression in basal epithelial cells is essential to determine which axis these cells are destined to: when AR level is low, their proliferation is promoted and may facilitate selfrenewal/proliferation of stem/progenitor cells, but when the AR levels increase, their destination is toward differentiation. These circumstances and the driving forces of AR expression toward proliferation or differentiation remain unclear.

After androgen deprivation via castration/androgen deprivation therapy (ADT), in normal prostate tissues, an extensive and rapid cell loss was observed, which appeared to affect mainly the luminal cells. In the process of prostate regeneration, induced by testosterone administration, it was observed that the luminal compartment rapidly regained its normal thickness with keratin expression pattern 

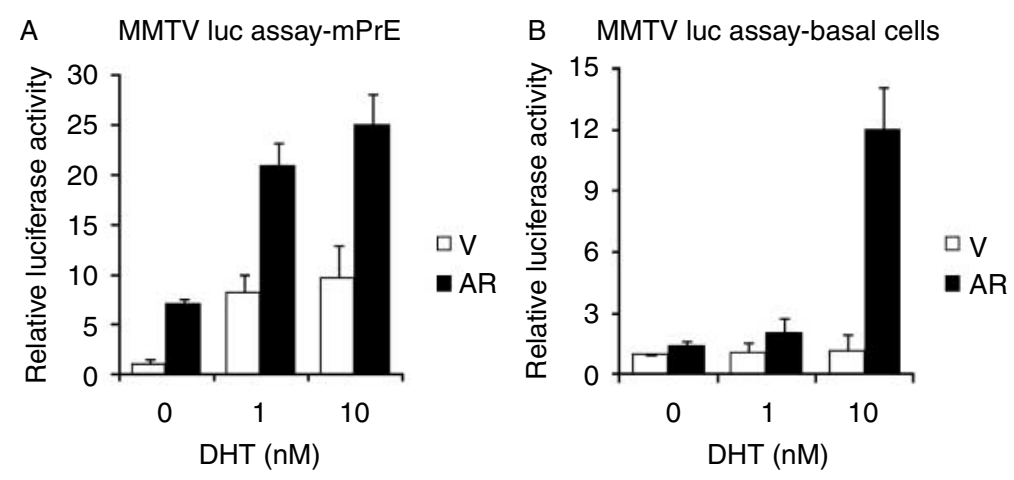

C

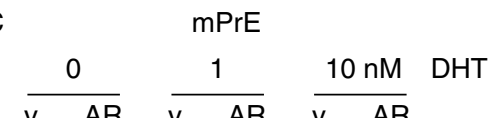

D
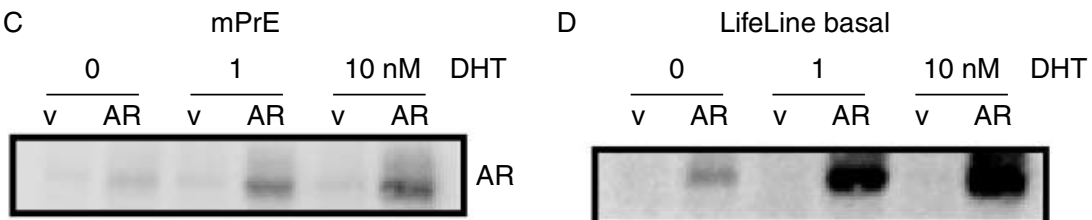

AR
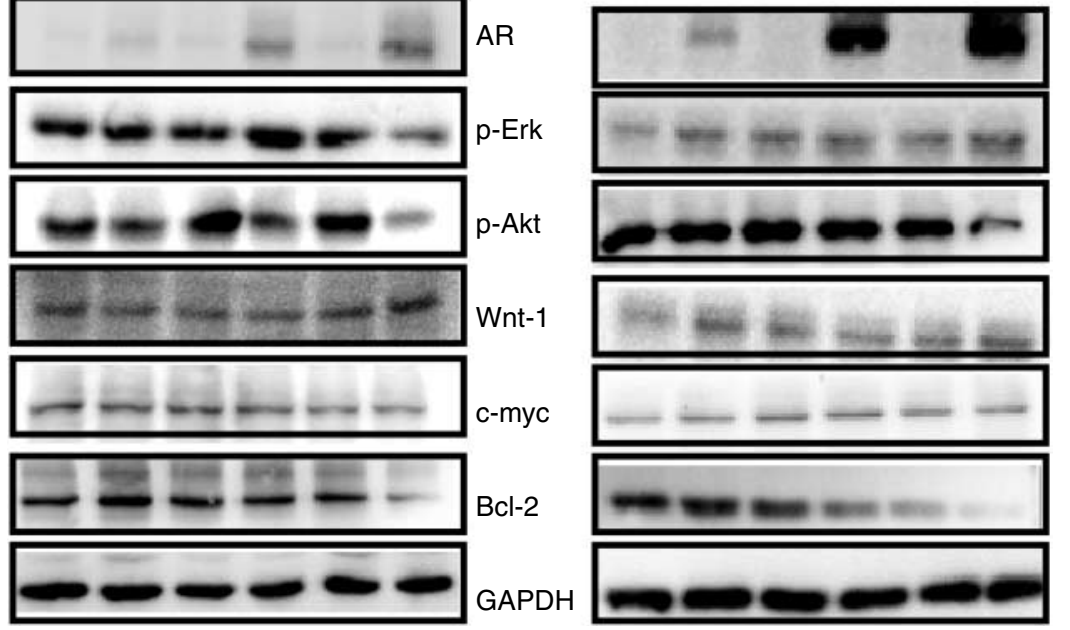

$\mathrm{Bcl}-2$

GAPDH

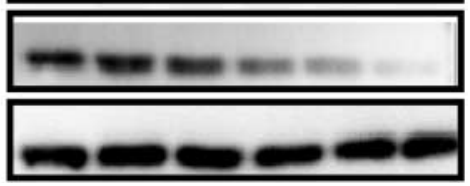

Figure 4 AR expression decreases signals related to cell growth/proliferation/survival in $\mathrm{mPrE}$ and Lifeline-basal cells. (A) $\mathrm{mPrE}$ and (B) Lifeline-basal cells were infected with lentivirus carrying AR or vector and transfected with MMTV-luciferase containing ARE. After transfection, cells were grown in three different androgenic concentrations $(0,1$, and $10 \mathrm{nM})$ and luciferase activity was measured by dual-luciferase assay (Promega) according to the manufacturer's manual. pRL-TK was used as internal control. (C) mPrE and (D) Lifeline-basal cells were prepared similar to A and B, and the expression levels of the indicated signal molecules were analyzed by western blot analysis.

intermediate between basal $\left(\mathrm{CK} 5^{+} / \mathrm{CK} 8^{-} / \mathrm{AR}^{\text {low }}\right)$ and luminal $\left(\mathrm{CK}^{-} / \mathrm{CK}^{\text {low }} / \mathrm{AR}^{\text {high }}\right)$ cells (Verhagen et al. 1988). In prostate tumors, the intermediate-type cells $\left(\mathrm{CK} 5^{\text {low }} / \mathrm{CK} 8^{\text {high }} / \mathrm{AR}^{\text {low }}\right)$ were also found increased after ADT (Verhagen et al. 1992, Schalken 2004, Niu et al. 2008). These results also support our hypothesis that the AR expression drives cells into differentiation to generate intermediate cells and into terminal differentiated luminal cells. However, the mechanism by which the AR expression in basal cells and progenitor cells is increased upon castration/ ADT is unclear. It was suggested that AR can become transactivated in the low-androgen environment of castration/ADT through a number of different mechanisms, including amplification and mutation of the receptor, cross talk with other signaling pathways, and altered regulation by coregulatory proteins (Lamont \& Tindall 2011). Recently, it has been shown that an enhancer in the AR second intron contributes to increased AR expression at low androgen levels (Cai et al. 2011). Therefore, we can speculate that these factors might contribute to the increased level of AR in those cell types and the subsequent differentiation process.

The suppressive role of AR in progenitor cells and basal cells can be applied to PCa therapeutic approach. Much recent evidence indicates that $\mathrm{PCa}$ contains stem/progenitor cells that might play essential roles in prostate tumorigenicity and metastasis; therefore, the therapeutic strategies targeting these cells are emerging (Maitland \& Collins 2008, Klarmann et al. 2009, Pfeiffer \& Schalken 2009, Li \& Tang 2011). 
We speculate that the suppressor role of AR can be applied to the development of new therapies to target self-renewal/ proliferation of stem/progenitor cells and the basal cells as we observed in this study that introducing AR could block selfrenewal/proliferation of these cells. As an introduction of AR into the cells is not practically possible, we might be able to develop a therapy targeting the downstream signaling molecules we revealed in these studies.

\section{Supplementary data}

This is linked to the online version of the paper at http://dx.doi.org/10.1530/ JOE-11-0474.

\section{Declaration of interest}

The authors declare that there is no conflict of interest that could be perceived as prejudicing the impartiality of the research reported.

\section{Funding}

This work was supported by NIH grants (CA122840 and CA127300) and Taiwan Department of Health Clinical Trial and Research Center of Excellence (DOH99-TD-B-111-004) to China Medical University, Taiwan.

\section{References}

Altuwaijri S, Wu CC, Niu YJ, Mizokami A, Chang HC \& Chang C 2007 Expression of human AR CDNA driven by its own promoter results in mild promotion, but not suppression, of growth in human prostate cancer PC-3 cells. Asian Journal of Andrology 9 181-188. (doi:10.1111/j.1745-7262.2007.00258.x)

Berger R, Febbo PG, Majumder PK, Zhao JJ, Mukherjee S, Signoretti S, Campbell KT, Sellers WR, Roberts TM, Loda M et al. 2004 Androgeninduced differentiation and tumorigenicity of human prostate epithelial cells. Cancer Research 64 8867-8875. (doi:10.1158/0008-5472.CAN-04-2938)

Bonkhoff H, Stein U \& Remberger K 1994 The proliferative function of basal cells in the normal and hyperplastic human prostate. Prostate 24 114-118. (doi:10.1002/pros.2990240303)

Bonkhoff H, Fixemer T \& Remberger K 1998 Relation between Bcl-2, cell proliferation, and the androgen receptor status in prostate tissue and precursors of prostate cancer. Prostate 34 251-258. (doi:10.1002/ (SICI)1097-0045(19980301)34:4 < 251::AID-PROS2>3.0.CO;2-K)

Cai C, He HH, Chen S, Coleman I, Wang H, Fang Z, Nelson PS, Liu XS, Brown M \& Balk SP 2011 Androgen receptor gene expression in prostate cancer is directly suppressed by the androgen receptor through recruitment of lysine-specific demethylase 1. Cancer Cell 20 457-471. (doi:10.1016/j. ccr.2011.09.001)

Garraway LA, Lin D, Signoretti S, Waltregny D, Dilks J, Bhattacharya N \& Loda M 2003 Intermediate basal cells of the prostate: in vitro and in vivo characterization. Prostate 55 206-218. (doi:10.1002/pros.10244)

Gurel B, Ali TZ, Montgomery EA, Begum S, Hicks J, Goggins M, Eberhart CG, Clark DP, Bieberich CJ, Epstein JI et al. 2010 NKX3.1 as a marker of prostatic origin in metastatic tumors. American Journal of Surgical Pathology 34 1097-1105. (doi:10.1097/PAS.0b013e3181e6cbf3)

Hayward SW, Baskin LS, Haughney PC, Cunha AR, Foster BA, Dahiya R, Prins GS \& Cunha GR 1996 Epithelial development in the rat ventral prostate, anterior prostate and seminal vesicle. Acta Anatomica 155 81-93. (doi:10.1159/000147793)
Johnson MA, Hernandez I, Wei Y \& Greenberg N 2000 Isolation and characterization of mouse probasin: an androgen-regulated protein specifically expressed in the differentiated prostate. Prostate 43 255-262. (doi:10.1002/ 1097-0045(20000601)43:4<255::AID-PROS4>3.0.CO;2-M)

Klarmann GJ, Hurt EM, Mathews LA, Zhang X, Duhagon MA, Mistree T, Thomas SB \& Farrar WL 2009 Invasive prostate cancer cells are tumor initiating cells that have a stem cell-like genomic signature. Clinical $\&$ Experimental Metastasis 26 433-446. (doi:10.1007/s10585-009-9242-2)

Lai JJ, Lai KP, Chuang KH, Chang P, Yu IC, Lin WJ \& Chang C 2009 Monocyte/macrophage androgen receptor suppresses cutaneous wound healing in mice by enhancing local TNF- $\alpha$ expression. Journal of Clinical Investigation 119 3739-3751. (doi:10.1172/JCI39335)

Lamont KR \& Tindall DJ 2011 Minireview: alternative activation pathways for the androgen receptor in prostate cancer. Molecular Endocrinology 25 897-907. (doi:10.1210/me.2010-0469)

Li H \& Tang DG 2011 Prostate cancer stem cells and their potential roles in metastasis. Journal of Surgical Oncology 103 558-562. (doi:10.1002/jso.21806)

Liao CP, Liang M, Cohen MB, Flesken-Nikitin A, Jeong JH, Nikitin AY \& Roy-Burman P 2010 Mouse prostate cancer cell lines established from primary and postcastration recurrent tumors. Hormones \& Cancer 1 44-54. (doi:10.1007/s12672-009-0005-y)

Ling MT, Chan KW \& Choo CK 2001 Androgen induces differentiation of a human papillomavirus $16 \mathrm{E} 6 / \mathrm{E} 7$ immortalized prostate epithelial cell line. Journal of Endocrinology 170 287-296. (doi:10.1677/joe.0.1700287)

Liu A, Wei L, Gardner WA, Deng CX \& Man YG 2009 Correlated alterations in prostate basal cell layer and basement membrane. International Journal of Biological Sciences 5 276-285. (doi:10.7150/ijbs.5.276)

Maitland NJ \& Collins AT 2008 Prostate cancer stem cells: a new target for therapy. Journal of Clinical Oncology 26 2862-2870. (doi:10.1200/JCO.2007. 15.1472)

Nishi N, Matuo Y, Nakamoto T \& Wada F 1988 Proliferation of epithelial cells derived from rat dorsolateral prostate in serum-free primary cell culture and their response to androgen. In Vitro Cellular \& Developmental Biology 24 778-786. (doi:10.1007/BF02623648)

Niu Y, Altuwaijri S, Lai KP, Wu CT, Ricke WA, Messing EM, Yao J, Yeh S \& Chang C 2008 Androgen receptor is a tumor suppressor and proliferator in prostate cancer. PNAS 105 12182-12187. (doi:10.1073/pnas.0804700105)

Pfeiffer MJ \& Schalken JA 2009 Stem cell characteristics in prostate cancer cell lines. European Urology 57 246-254. (doi:10.1016/j.eururo.2009.01.015)

Prins GS, Birch L \& Greene GL 1991 Androgen receptor localization in different cell types of the adult rat prostate. Endocrinology 129 3187-3199. (doi:10.1210/endo-129-6-3187)

Schalken JA 2004 Molecular and cellular prostate biology: origin of prostatespecific antigen expression and implications for benign prostatic hyperplasia. BJU International 93 (Suppl 1) 5-9. (doi:10.1111/j.1464-410X.2003.04633.x)

Simanainen U, Allan CM, Lim P, McPherson S, Jimenez M, Zajac JD, Davey RA \& Handelsman DJ 2007 Disruption of prostate epithelial androgen receptor impedes prostate lobe-specific growth and function. Endocrinology 148 2264-2272. (doi:10.1210/en.2006-1223)

Simanainen U, McNamara K, Gao YR \& Handelsman DJ 2009 Androgen sensitivity of prostate epithelium is enhanced by postnatal androgen receptor inactivation. American Journal of Physiology. Endocrinology and Metabolism 296 E1335-E1343. (doi:10.1152/ajpendo.00017.2009)

Soriano P 1999 Generalized lacZ expression with the ROSA26 Cre reporter strain. Nature Genetics 21 70-71. (doi:10.1038/5007)

Titus MA, Gregory CW, Ford OH III, Schell MJ, Maygarden SJ \& Mohler JL $2005 a$ Steroid $5 \alpha$-reductase isozymes I and II in recurrent prostate cancer. Clinical Cancer Research 11 4365-4371. (doi:10.1158/1078-0432.CCR-04-0738)

Titus MA, Schell MJ, Lih FB, Tomer KB \& Mohler JL 2005b Testosterone and dihydrotestosterone tissue levels in recurrent prostate cancer. Clinical Cancer Research 11 4653-4657. (doi:10.1158/1078-0432.CCR-05-0525)

Uzgare AR, Xu Y \& Isaacs JT 2004 In vitro culturing and characteristics of transit amplifying epithelial cells from human prostate tissue. Journal of Cellular Biochemistry 91 196-205. (doi:10.1002/jcb.10764)

Verhagen AP, Aalders TW, Ramaekers FC, Debruyne FM \& Schalken JA 1988 Differential expression of keratins in the basal and luminal compartments of rat prostatic epithelium during degeneration and regeneration. Prostate 13 25-38. (doi:10.1002/pros.2990130104) 
Verhagen AP, Ramaekers FC, Aalders TW, Schaafsma HE, Debruyne FM \& Schalken JA 1992 Colocalization of basal and luminal cell-type cytokeratins in human prostate cancer. Cancer Research 52 6182-6187.

Wang Y, Hayward SW, Donjacour AA, Young P, Jacks T, Sage J, Dahiya R, Cardiff RD, Day ML \& Cunha GR 2000 Sex hormone-induced carcinogenesis in Rb-deficient prostate tissue. Cancer Research 60 6008-6017.

Whitacre DC, Chauhan S, Davis T, Gordon D, Cress AE \& Miesfeld RL 2002 Androgen induction of in vitro prostate cell differentiation. Cell Growth $\mathcal{E}$ Differentiation 13 1-11.

Wu CT, Altuwaijri S, Ricke WA, Huang SP, Yeh S, Zhang C, Niu Y, Tsai MY \& Chang C 2007 Increased prostate cell proliferation and loss of cell differentiation in mice lacking prostate epithelial androgen receptor. PNAS 104 12679-12684. (doi:10.1073/pnas.0704940104)

Xue Y, Smedts F, Debruyne FM, de la Rosette JJ \& Schalken JA 1998 a Identification of intermediate cell types by keratin expression in the developing human prostate. Prostate 34 292-301. (doi:10.1002/ (SICI)1097-0045(19980301)34:4<292::AID-PROS7>3.0.CO;2-J)
Xue Y, Smedts F, Verhofstad A, Debruyne F, de la Rosette J \& Schalken J $1998 b$ Cell kinetics of prostate exocrine and neuroendocrine epithelium and their differential interrelationship: new perspectives. Prostate. Supplement 8 62-73. (doi:10.1002/(SICI)1097-0045(1998)8 + <62::AIDPROS10 $>3.0 . \mathrm{CO} ; 2-\mathrm{P})$

Yeh S, Tsai MY, Xu Q, Mu XM, Lardy H, Huang KE, Lin H, Yeh SD, Altuwaijri S, Zhou X et al. 2002 Generation and characterization of androgen receptor knockout (ARKO) mice: an in vivo model for the study of androgen functions in selective tissues. PNAS 99 13498-13503. (doi:10.1073/pnas.212474399)

Received in final form 24 February 2012

Accepted 5 March 2012

Made available online as an Accepted Preprint 5 March 2012 